\title{
Image analysis
}

\section{Handling of lymphocyte dense images}

For WSIs with a huge lymphocytic count, we suggest to reduce the search space for possible infiltrates using heatmaps consisting of tiles $(16.19 \mu \mathrm{m} \times 16.19 \mu \mathrm{m})$, which represent a sum of $\mathrm{CD}^{+}$and $\mathrm{CD} 20^{+}$cells density maps.

\section{Nuclei detection}

We used an algorithm presented in [1]. The key idea of the approach is to take advantage of well-differentiated objects in each slide to learn about the appearance of the tissue and in particular about the appearance of low-differentiated objects. Well-differentiated objects are automatically selected from representative regions, then slide-specific visual context models [2] are learned, and finally the resulting posterior maps are used to perform the final detection steps on the WSI.

\section{References}

[1] Brieu N, Pauly O, Zimmermann J, Binnig G, Schmidt G. Slide specific models for segmentation of differently stained digital histopathology whole slide images. Proc SPIE. 2016;9784:978410-978417.

[2] Criminisi A, Shotton J, Bucciarelli S. Decision Forests with Long-Range Spatial Context for Organ Localization in CT Volumes. In: MICCAI-PMMIA; 2009. 